\title{
Chromatic number and complete graph substructures for degree sequences
}

\author{
Zdeněk Dvořák ${ }^{* \dagger}$ \\ Department of Mathematics \\ Simon Fraser University \\ Burnaby, B.C. V5A 1S6 \\ email: rakdver@kam.mff.cuni.cz
}

\author{
Bojan Mohar $\$ \S$ \\ Department of Mathematics \\ Simon Fraser University \\ Burnaby, B.C. V5A 1S6 \\ email: mohar@sfu.ca
}

June 4, 2018

\begin{abstract}
Given a graphic degree sequence $D$, let $\chi(D)$ (respectively $\omega(D)$, $h(D)$, and $H(D)$ ) denote the maximum value of the chromatic number (respectively, the size of the largest clique, largest clique subdivision, and largest clique minor) taken over all simple graphs whose degree sequence is $D$. It is proved that $\chi(D) \leq h(D)$. Moreover, it is shown that a subdivision of a clique of order $\chi(D)$ exists where each edge is subdivided at most once and the set of all subdivided edges forms a collection of disjoint stars. This bound is an analogue of the Hajós Conjecture for degree sequences and, in particular, settles a conjecture of Neil Robertson that degree sequences satisfy the bound $\chi(D) \leq$ $H(D)$ (which is related to the Hadwiger Conjecture). It is also proved that $\chi(D) \leq \frac{6}{5} \omega(D)+\frac{3}{5}$ and that $\chi(D) \leq \frac{4}{5} \omega(D)+\frac{1}{5} \Delta(D)+1$, where $\Delta(D)$ denotes the maximum degree in $D$. The latter inequality is a strengthened version of a conjecture of Bruce Reed. All derived inequalities are best possible.
\end{abstract}

Keywords: Degree sequence, maximum clique, clique minor, clique subdivision, Hajos Conjecture, Hadwiger Conjecture, Reed Conjecture.

\footnotetext{
* Supported in part through a postdoctoral position at Simon Fraser University and by the grant GA201/09/0197 of Czech Science Foundation.

${ }^{\dagger}$ On leave from: Institute of Theoretical Informatics, Charles University, Prague, Czech Republic.

${ }^{\ddagger}$ Supported in part by an NSERC Discovery Grant (Canada), by the Canada Research Chair program, and by the Research Grant P1-0297 of ARRS (Slovenia).

${ }^{\S}$ On leave from: IMFM \& FMF, Department of Mathematics, University of Ljubljana, Ljubljana, Slovenia.
} 


\section{Introduction}

All graphs considered in this paper are simple, without loops or parallel edges. A multiset of non-negative integers, usually written in the form of a non-increasing sequence $d_{1} \geq d_{2} \geq \cdots \geq d_{n}$, is called a graphic degree sequence if there exists a simple graph $G$ of order $n$ whose vertex degrees are $d_{1}, d_{2}, \ldots, d_{n}$. Given a graph $G$, we denote by $D(G)$ its degree sequence, and given a degree sequence $D$, we let

$$
\mathcal{R}(D)=\{G \mid D(G)=D\}
$$

denote the set of all realizations of $D$. By $\delta(D)$ and $\Delta(D)$ we denote the minimum and the maximum degree of $D$, respectively.

S. B. Rao introduced the following ordering for degree sequences: $D \preceq D^{\prime}$ if there exist $G \in \mathcal{R}(D)$ and $G^{\prime} \in \mathcal{R}\left(D^{\prime}\right)$ such that $G$ is an induced subgraph of $G^{\prime}$. Motivated by the progress made by Robertson and Seymour [9] on the well-quasi-ordering of graphs ordered by the graph minors relation, he proposed the following conjecture.

Conjecture 1.1 (S. B. Rao, 1981). The degree sequences are well-quasiordered with respect to the relation $\preceq$.

Recently, Chudnovsky and Seymour [3] announced a proof of this conjecture.

Let us introduce the following notation. If $D$ is a degree sequence, we let $\chi(D)$ (respectively $\omega(D), h(D)$, and $H(D)$ ) denote the maximum value of the chromatic number (respectively, the size of the largest clique, largest clique subdivision, and largest clique minor) taken over all graphs in $\mathcal{R}(D)$. Let us observe that $\omega(D) \leq h(D) \leq H(D)$.

Motivated by Rao's conjecture, Neil Robertson proposed a conjecture on degree sequences that is a relaxation of the famous Hadwiger Conjecture claiming that every graph with chromatic number $k$ contains a $k$-clique as a minor. Despite many attempts, the Hadwiger Conjecture remains open, thus its relaxations are of high interest.

Conjecture 1.2 (Robertson [8]). For every graphic degree sequence $D$, we have $\chi(D) \leq H(D)$.

In a recent work, Robertson and Song [10] proved a special case of Conjecture 1.2 when the degree sequence contains at most two distinct degree values.

In this paper we consider a stronger version of Conjecture 1.2 that is related to the Hajós Conjecture: 
Conjecture 1.3. For every graphic degree sequence $D$, we have $\chi(D) \leq$ $h(D)$.

This conjecture is particularly interesting, not only because it strengthens Conjecture 1.2, but also because the Hajós Conjecture for graphs fails (Catlin [2]; see also [11, 12]).

The main result of our work is a proof of Conjecture 1.3 (see Theorems 4.1 and 3.5). It is shown that this conjecture holds in a quite strong way. Namely, we prove that there is a graph $G \in \mathcal{R}(D)$ containing a subdivided complete graph of order $\chi(D)$ such that each edge is subdivided at most once and the set of all subdivided edges forms a collection of disjoint stars. This, in particular, settles Conjectures 1.2 and 1.3 .

We also address a question how close to $\chi(D)$ is the maximum clique number $\omega(D)$. We prove (cf. Theorem 3.2) that

$$
\chi(D) \leq \frac{6}{5} \omega(D)+\frac{3}{5}
$$

holds for every degree sequence $D$. If $\chi(D) \leq \frac{1}{2} n(D)$, where $n(D)$ is the number of vertices for the degree sequence $D$, and if $\delta(D) \geq \chi(D)-1$, then one can prove that $\omega(D)=\chi(D)$. However, the situation changes when $\chi(D)>\frac{1}{2} n(D)$. For example, any realization of the degree sequence $D=(5 k-3)^{5 k}$ (i.e., $d_{i}=5 k-3$ for $\left.i=1, \ldots, 5 k\right)$ is a complement of a union of cycles, thus it has $\chi(D)=3 k$ (realized by a join of 5 -cycles) and $\omega(D)=\left\lfloor\frac{5 k}{2}\right\rfloor$ (realized by a complement of a $5 k$-cycle). If $k$ is odd, then $\chi(D)=\frac{6}{5} \omega(D)+\frac{3}{5}$. This example shows that the inequality (11) is best possible.

Finally, we consider an analogue of Reed's Conjecture [7] (cf. Section 3) bounding the chromatic number by a convex combination of the clique number and the maximum degree. Our Theorem 3.4 shows that

$$
\chi(D) \leq \frac{4}{5} \omega(D)+\frac{1}{5} \Delta(D)+1 .
$$

This bound is best possible in the sense that equality holds for infinitely many graphic sequences $D$ and that for every $\alpha>\frac{4}{5}$ and every $\beta$, there exists a degree sequence $D$ such that

$$
\chi(D)>\alpha \omega(D)+(1-\alpha) \Delta(D)+\beta .
$$

\section{Preliminary results}

Let us recall the following folklore results about graphic degree sequences, cf., e.g. [1]. 
Lemma 2.1. There exists a tree with degree sequence $d_{1} \geq d_{2} \geq \cdots \geq d_{n}$ if and only if $d_{n} \geq 1$ and $\sum_{i=1}^{n} d_{i}=2 n-2$.

Lemma 2.2. Let $a_{1} \geq a_{2} \geq \cdots \geq a_{n}$ and $b_{1} \geq b_{2} \geq \cdots \geq b_{m}$ be two sequences of positive integers such that $a_{1} \leq m, \sum_{i=1}^{n} a_{i}=\sum_{i=1}^{m} b_{i}$ and $b_{1} \leq b_{m}+1$. Then there exists a bipartite graph $G$ with parts $u_{1}, u_{2}, \ldots, u_{n}$ and $v_{1}, v_{2}, \ldots, v_{m}$ such that the degree of $u_{i}$ is $a_{i}$ for $1 \leq i \leq n$ and the degree of $v_{i}$ is $b_{i}$ for $1 \leq i \leq m$.

Let us prove a slightly stronger statement:

Lemma 2.3. Let $a_{1} \geq a_{2} \geq \cdots \geq a_{n}$ and $b_{1} \geq b_{2} \geq \cdots \geq b_{m}$ be two sequences of positive integers such that $n \leq m, a_{1} \leq m, \sum_{i=1}^{n} a_{i}=\sum_{i=1}^{m} b_{i}$, and $b_{1} \leq b_{m}+1$. Let $A=\left\{u_{1}, u_{2}, \ldots, u_{n}\right\}$ and $B=\left\{v_{1}, v_{2}, \ldots, v_{m}\right\}$ be two sets of vertices. Then there exists a bipartite graph $G$ with parts $A$ and $B$ such that $G$ has a matching covering $A$, the degree of $u_{i}$ is $a_{i}$ for $1 \leq i \leq n$ and the degree of $v_{i}$ is $b_{i}$ for $1 \leq i \leq m$.

Proof. We prove the claim by induction. The lemma holds if $n=1$, as in that case $m \geq a_{1}=\sum_{i=1}^{m} b_{i} \geq m$, thus $a_{1}=m$ and $b_{1}=b_{2}=\cdots=b_{m}=1$, and we take $G=K_{1, m}$. Suppose now that $n \geq 2$ and that the claim is true for all sequences $a_{1}^{\prime}, \ldots, a_{n^{\prime}}^{\prime}$ and $b_{1}^{\prime}, \ldots, b_{m^{\prime}}^{\prime}$ such that $n^{\prime}<n$. If $b_{1}=1$, then $G$ is a union of stars, thus assume that $b_{1} \geq 2$. This implies that $a_{1} \geq 2$, as if $a_{1}=1$, then $m \geq n=\sum_{i=1}^{n} a_{i}=\sum_{i=1}^{m} b_{i} \geq m$ and $b_{1}$ would be equal to 1 . On the other hand, $m n \geq \sum_{i=1}^{n} a_{i}=\sum_{i=1}^{m} b_{i} \geq m\left(b_{1}-1\right)+1$, thus $b_{1} \leq n$.

Consider the sequences $a_{2}-1, a_{3}-1, \ldots, a_{b_{1}}-1, a_{b_{1}+1}, \ldots, a_{n}$ and $b_{2}-$ $1, b_{3}-1, \ldots, b_{a_{1}}-1, b_{a_{1}+1}, \ldots, b_{n}$. Let $a_{1}^{\prime} \geq a_{2}^{\prime} \geq \cdots \geq a_{n^{\prime}}^{\prime}$ and $b_{1}^{\prime} \geq$ $b_{2}^{\prime} \geq \cdots \geq b_{m^{\prime}}^{\prime}$ be the positive elements of these two sequences. If the first sequence is empty, then $a_{2}=a_{3}=\cdots=a_{n}=1$ and $n=b_{1}$, and hence $m+b_{1}-1 \geq a_{1}+b_{1}-1=\sum_{i=1}^{n} a_{i}=\sum_{i=1}^{m} b_{i} \geq b_{1}+m-1$. Therefore equalities hold, and this is only possible when $a_{1}=m$ and $b_{2}=b_{3}=\cdots=b_{m}=1$. It follows that $n=b_{1}=2$ and $a_{2}=1$. In this case $G$ is $K_{1, m}$ with one edge subdivided, which has a matching covering $A$ as $m \geq n=2$.

Therefore, we may assume that $n^{\prime} \geq 1$. Note that $\sum_{i=1}^{n^{\prime}} a_{i}^{\prime}=\sum_{i=1}^{m^{\prime}} b_{i}^{\prime}$, which implies that $m^{\prime} \geq 1$. Also, observe that $b_{1}^{\prime} \leq b_{m^{\prime}}^{\prime}+1$. Suppose first that $m^{\prime}<m-1$, i.e., $b_{a_{1}}=1$, and thus $b_{1}=2$. If $a_{2}>1$, then $n^{\prime}=n-1$, and let $G$ be the graph obtained from the union of stars $K_{1, a_{1}^{\prime}}, K_{1, a_{2}^{\prime}}, \ldots$, $K_{1, a_{n^{\prime}}^{\prime}}$ by adding the vertices of $u_{1}$ and $v_{1}$ of degrees $a_{1}$ and $b_{1}$, respectively (with $u_{1} v_{1}$ in the matching), joined to the appropriate vertices of the stars. If $a_{2}=1$, then $G$ is a union of a matching and the star $K_{1, a_{1}}$ with some (but not all) edges subdivided. Therefore, we may assume from now on that $m^{\prime}=m-1$. 
Suppose now that $n^{\prime}<n-1$, i.e., $a_{b_{1}}=1$. In that case,

$$
\begin{aligned}
\left(b_{1}-2\right) m-b_{1}+n+a_{b_{1}-1}+1 & \geq\left(b_{1}-2\right) a_{1}+a_{b_{1}-1}+n-b_{1}+1 \\
& \geq \sum_{i=1}^{n} a_{i}=\sum_{i=1}^{m} b_{i} \geq\left(b_{1}-1\right) m+1 .
\end{aligned}
$$

Thus $a_{b_{1}-1} \geq m-n+b_{1} \geq 2$, hence $n^{\prime}=n-2$. The sequences $a_{1}-1, a_{1}^{\prime}$, $a_{2}^{\prime}, \ldots, a_{n^{\prime}}^{\prime}$ and $b_{2}, b_{3}, \ldots, b_{m}$ satisfy the assumptions of the lemma; let $G^{\prime}$ be the graph corresponding to them. We let $G$ be the graph obtained from $G^{\prime}$ by adding the vertices $u_{b_{1}}$ and $v_{1}$ and joining $v_{1}$ with $u_{1}, u_{2}, \ldots, u_{b_{1}}$. The edge $u_{b_{1}} v_{1}$ is added to the matching covering $A$.

Finally, consider the case when $n^{\prime}=n-1$ and $m^{\prime}=m-1$. Note that $n^{\prime} \leq m^{\prime}$. If $b_{1}=n$, then $a_{1}^{\prime}=a_{2}-1 \leq a_{1}-1 \leq m^{\prime}$. On the other hand, if $b_{1}<n$, then $\left(b_{1}+1\right) a_{b_{1}+1} \leq \sum_{i=1}^{n} a_{i}=\sum_{i=1}^{m} b_{i} \leq m b_{1}$, and thus $a_{b_{1}+1} \leq m-1$ and again, $a_{1}^{\prime} \leq m^{\prime}$. Therefore, the sequences $a_{1}^{\prime}, \ldots, a_{n^{\prime}}^{\prime}$ and $b_{1}^{\prime}, \ldots, b_{m^{\prime}}^{\prime}$ satisfy the assumptions of the lemma; let $G^{\prime}$ be the graph corresponding to them. We let $G$ be the graph obtained from $G^{\prime}$ by adding the vertices $u_{1}$ and $v_{1}$ and joining $u_{1}$ with $v_{1}, \ldots, v_{a_{1}}$ and $v_{1}$ with $u_{2}, \ldots$, $u_{b_{1}}$. The edge $u_{1} v_{1}$ is added to the matching covering $A$.

We shall also need the following simple observation:

Lemma 2.4. There exists a graph $G$ with $n \geq 1$ vertices, e edges and $1 \leq \delta(G) \leq \Delta(G) \leq 2$ if and only if $e=n \geq 3$ or $e+1 \leq n \leq 2 e$ and $n \geq 2$.

Proof. Without loss of generality, if such a graph exists, then it is either a cycle, or a union of a (possibly empty) matching and a path of length at least one. The former is possible if and only if $e=n \geq 3$. The latter is possible if and only if $e+1 \leq n \leq 2 e$ and $n \geq 2$.

Rao [6] proved the following:

Theorem 2.5. A sequence $d_{1} \geq d_{2} \geq \cdots \geq d_{n}$ of nonnegative integers is a degree sequence of a graph $G$ with $\omega(G) \geq k$ if and only if $\sum_{i=1}^{n} d_{i}$ is even, $d_{k} \geq k-1$, and for $0 \leq s \leq k$ and $0 \leq t \leq n-k$,

$$
\begin{aligned}
\sum_{i=1}^{s} d_{i} & +\sum_{i=k+1}^{k+t} d_{i}-2\left(\begin{array}{c}
s+t \\
2
\end{array}\right) \\
& \leq \sum_{i=s+1}^{k} \min \left(s+t, d_{i}+s-k+1\right)+\sum_{i=k+t+1}^{n} \min \left(s+t, d_{i}\right) .
\end{aligned}
$$


Furthermore, if these conditions are satisfied, then we can choose $G$ so that the vertices of the clique of size $k$ have degrees $d_{1}, d_{2}, \ldots, d_{k}$.

Let us recall the characterization of graphic degree sequences by Erdös and Gallai [4] in the following form:

Theorem 2.6. A sequence $d_{1}, d_{2}, \ldots, d_{n}$ of non-negative integers is graphic if and only if $\sum_{i=1}^{n} d_{i}$ is even and for every $I \subseteq\{1, \ldots, n\}$,

$$
\sum_{i \in I} d_{i} \leq|I|(|I|-1)+\sum_{i \in\{1, \ldots, n\} \backslash I} \min \left(d_{i},|I|\right) .
$$

If $d_{1} \geq d_{2} \geq \cdots \geq d_{n}$, then property (3) can be checked only for subsets of the form $I=\{1,2, \ldots, t\}, 1 \leq t \leq n$.

Yin and Li [13, Theorem 1.8] showed the following:

Theorem 2.7. Suppose that a graphic sequence $d_{1} \geq \cdots \geq d_{n}$ satisfies the following conditions: $d_{k} \geq k-1, n \geq 2 k$, and $d_{2 k} \geq k-2$. Then it satisfies the assumptions of Theorem 2.5.

We will need the following variation:

Lemma 2.8. A graphic sequence $d_{1} \geq \cdots \geq d_{2 k-1}$ of length $2 k-1$ with $d_{2 k-1} \geq k-1$ satisfies the assumptions of Theorem 2.5 if and only if

$$
\sum_{i=1}^{k-1}\left(d_{i}-d_{k}\right)+\sum_{i=k+1}^{2 k-1}\left(d_{k}-d_{i}\right) \geq 2 k-2-d_{k} .
$$

Proof. Consider the condition (2) of Theorem 2.5 with $s=0$ and $t=k-1$ :

$$
\sum_{i=k+1}^{2 k-1} d_{i}-2\left(\begin{array}{c}
k-1 \\
2
\end{array}\right) \leq \sum_{i=1}^{k} \min \left(k-1, d_{i}-k+1\right) .
$$

As $d_{i} \leq 2 k-2$, we have $\min \left(k-1, d_{i}-k+1\right)=d_{i}-k+1$ for $1 \leq i \leq k$. Subtracting $k d_{k}$ from both sides of (5), we get

$$
\sum_{i=k+1}^{2 k-1}\left(d_{i}-d_{k}\right)-d_{k}-(k-1)(k-2) \leq \sum_{i=1}^{k-1}\left(d_{i}-d_{k}\right)-k(k-1),
$$

which implies (4).

The above derivation actually shows that (41) and (5) are equivalent. Therefore, we need to prove that (4) (or (5) ) implies the condition (2) of 
Theorem 2.5 for the choices of $s$ and $t$ such that $0 \leq s \leq k, 0 \leq t \leq k-1$ and either $s \neq 0$ or $t \neq k-1$.

If $d_{k} \geq k+t-1$ and $s<k$, then $\min \left(s+t, d_{i}+s-k+1\right)=s+t=$ $\min \left(s+t, d_{i}\right)$ for $s+1 \leq i \leq k$. Since the sequence $d_{1}, \ldots, d_{n}$ is graphic, (2) follows from (3) with $I=\{1,2, \ldots, s, k+1, k+2, \ldots, k+t\}$. The same argument works if $s=k$ (independently of the value of $d_{k}$ ), since the first sum on the right hand side of (2) vanishes. Therefore, we may henceforth assume that $d_{k} \leq k+t-2$ and $s \leq k-1$.

As $d_{1} \leq 2 k-2, d_{2 k-1} \geq k-1, d_{i} \geq d_{k}$ for $i \leq k$ and $d_{i} \leq d_{k}$ for $i \geq k$, it suffices to show that

$$
\begin{aligned}
s(2 k-2)+t d_{k}-2\left(\begin{array}{c}
s+t \\
2
\end{array}\right) \leq & (k-s) \min \left(s+t, d_{k}+s-k+1\right)+ \\
& (k-1-t) \min (s+t, k-1) .
\end{aligned}
$$

As $d_{k}+s-k+1 \leq s+t$, the inequality (6) is equivalent to

$$
\begin{aligned}
(k+s)(k-1)+(s+t-k) d_{k} \leq & (s+t)(s+t-1)+s(k-s)+ \\
& (k-t-1) \min (s+t, k-1) .
\end{aligned}
$$

Let us first assume that $s+t \geq k$. Since $d_{k} \leq k+t-2$, it suffices to prove that

$(k+s)(k-1)+(s+t-k)(k+t-2) \leq(s+t)(s+t-1)+s(k-s)+(k-t-1)(k-1)$,

which is equivalent to $(k-t-2)(k-s-2)+k-3 \geq 0$. If $t=k-1$, then $(k-t-2)(k-s-2)+k-3=s-1 \geq 0($ as $s+t \geq k)$. Similarly, if $s=k-1$, then $(k-t-2)(k-s-2)+k-3=t-1 \geq 0$. If $s \leq k-2$ and $t \leq k-2$, then $k \leq s+t \leq 2 k-4$, hence $k \geq 4$ and $(k-t-2)(k-s-2)+k-3 \geq 1$, thus the condition is satisfied.

Let us now consider the remaining case when $s+t \leq k-1$. As $\min (s+$ $t, k-1)=s+t, d_{k} \geq k-1$, and the coefficient of $d_{k}$ on the left hand side of (7) is negative, it suffices to prove that

$(k+s)(k-1) \leq(k-s-t)(k-1)+(s+t)(s+t-1)+s(k-s)+(k-t-1)(s+t)$.

This is equivalent to $t \leq s t$. If $s>0$, then this condition is satisfied. So, it remains to consider the case when $s=0$. The condition (2) of Theorem 2.5 then becomes

$$
\sum_{i=k+1}^{k+t} d_{i} \leq t(k-2)+\sum_{i=1}^{k} \min \left(d_{i}-k+1, t\right),
$$


which is equivalent to

$$
t+\sum_{i=1}^{t}\left(d_{k+i}-k+1\right) \leq \sum_{i=1}^{k} \min \left(d_{i}-k+1, t\right) .
$$

Note that $d_{k+i}-k+1 \leq \min \left(d_{i+1}-k+1, t\right)$ since $d_{k+i}-k+1 \leq d_{k}-k+1 \leq t-1$ and $d_{k+i} \leq d_{i+1}$, for $1 \leq i \leq t$. Therefore, (8) holds if $d_{1} \geq k+t-1$. Suppose that $d_{1} \leq k+t-2$, i.e., $t \geq d_{1}-k+2$. Then $\min \left(d_{i}-k+1, t\right)=d_{i}-k+1$ for $1 \leq i \leq k$. Therefore, for $t \geq d_{1}-k+2$, the right-hand side of (8) is independent of $t$, and the left-hand side is non-decreasing in $t$, and hence the condition is satisfied for all $t$ if and only if it is satisfied for $t=k-1$, which is precisely our original assumption (5).

\section{Chromatic number and cliques}

For graphs $G_{1}, G_{2}, \ldots, G_{k}$, let $G_{1}+G_{2}+\ldots+G_{k}$ be their join, i.e., the graph obtained from the disjoint union of $G_{1}, G_{2}, \ldots, G_{k}$ by adding all edges between $V\left(G_{i}\right)$ and $V\left(G_{j}\right)$ for all $i, j$ such that $1 \leq i<j \leq k$. A graph $G$ is hypo-matchable if for each $v \in V(G), G-v$ has a perfect matching. A graph $G$ is $\chi$-critical if for each $v \in V(G), \chi(G-v)<\chi(G)$.

A graph $G$ is basic if

- $\chi(G) \leq \omega(D(G))$, or

- $G$ is $\chi$-critical, the number $n$ of vertices of $G$ is odd, $n=2 m+1$, $\chi(G)=m+1, \omega(D(G))=m$ and the complement of $G$ is hypomatchable.

We say that $G$ is nontrivial if $\chi(G)>\omega(D(G))$. Note that Lemma 2.8 describes the degree sequences of nontrivial basic graphs, that is, the degree sequences of nontrivial basic graphs do not satisfy the condition (44). The following lemma shows that when considering the behavior of $\chi(G)$ and $\omega(D(G))$, then we only care about the basic graphs.

Lemma 3.1. Any graph $G$ has an induced subgraph $G^{\prime}$ such that $\chi(G)=$ $\chi\left(G^{\prime}\right)$ and $G^{\prime}$ is a join of basic graphs.

Proof. For a contradiction, assume that $G$ is a smallest counterexample. Let $n$ be the number of vertices of $G$. As $G$ is not basic, $\chi(G) \geq \omega(D(G))+1$. As $G$ is a smallest counterexample, $G$ is $\chi$-critical, and thus $\delta(G) \geq \chi(G)-1 \geq$ $\omega(D(G))$. By Theorem 2.7, this implies that $n \leq 2 \omega(D(G))+1$. Also, $G$ is not a join of two graphs, i.e., the complement of $G$ is connected. 
Consider a coloring $\varphi$ of $G$ by $\chi(G)$ colors, such that the set of vertices $B$ that belong to color classes of size at least three is as small as possible. Let $k=|V(G-B)|$ and let $c$ be the number of color classes of $\varphi$ restricted to $G-B$. As $2 \chi(G) \geq 2 \omega(D(G))+2>n, 2 c>k$. Let $M$ be the set of color classes of $\varphi$ of size two. Note that $M$ is a maximum matching in the complementary graph $\overline{G-B}$ and that $|M|=k-c$. Conversely, any matching in $\overline{G-B}$ of size $k-c$ corresponds to a coloring of $G$ by $\chi(G)$ colors. Also, any vertex $v$ of $G-B$ that is not incident with $M$ is adjacent to all vertices of $B$, as otherwise if $v$ is not adjacent to a vertex $u \in B$, then we can set the color of $u$ to $\varphi(v)$, thus decreasing the size of $B$.

By the Edmonds-Gallai theorem on maximum matchings in graphs, there exists $T \subseteq V(G-B)$ and a matching $M^{\prime}$ in $\overline{G-B}$ such that each component of $\overline{G-B}-T$ is hypo-matchable, each edge of $M^{\prime}$ is incident with exactly one vertex of $T$, and no component of $\overline{G-B}-T$ is incident with more than one edge of $M^{\prime}$. Moreover, each vertex in $T$ is incident with an edge in $M^{\prime}$. Let $C$ be the set of components of $\overline{G-B}-T$. Let $t=|T|$ and let $h=|C|-t$ be the number of components of $\overline{G-B}-T$ that are not incident with an edge of $M^{\prime}$. Note that $h=2 c-k>0$. Consider the bipartite graph $H$ with parts $T$ and $C$, such that a vertex $u \in T$ and a component $K \in C$ are adjacent in $H$ if and only if there exists a vertex $v \in K$ such that $u$ and $v$ are non-adjacent in $G$. Let $C^{\prime} \subseteq C$ be the set of components that are covered by every matching in $H$ of size $t$. By Hall's theorem, there exists a set $T^{\prime} \subseteq T$ such that $\left|T^{\prime}\right|=\left|C^{\prime}\right|$ and the vertices of $T^{\prime}$ are adjacent in $G$ to all vertices of the components in $C \backslash C^{\prime}$. Consider now a component $K \in C \backslash C^{\prime}$ and a vertex $v \in K$. There exists a matching in $H$ of size $t$ that does not cover $K$, and this matching can be extended to a matching $M_{1}$ in $\overline{G-B}-T$ of size $k-c$ that does not cover $v$, as $K$ is hypo-matchable. It follows that $v$ is adjacent in $G$ to all vertices of $B$. Since the choice of $K$ and $v$ was arbitrary, it follows that all vertices of the components of $C \backslash C^{\prime}$ are adjacent to all vertices of $B$. Furthermore, note that for any component $K \in C \backslash C^{\prime}, \chi(G[V(K)])=(|V(K)|+1) / 2$. Also, $\left|C \backslash C^{\prime}\right| \geq h>0$.

Let $G_{0}$ be the subgraph of $G$ induced by $\bigcup_{K \in C^{\prime}} V(K) \cup B \cup T^{\prime}$, and let $G_{1}, G_{2}, \ldots, G_{a}$ be the subgraphs of $G$ induced by the vertex sets of the elements of $C \backslash C^{\prime}$. Let $G^{\prime}$ be the join of $G_{0}, G_{1}, \ldots, G_{a}$. Observe that $\chi\left(G^{\prime}\right)=\chi(G)$ and that $G^{\prime}$ is an induced subgraph of $G$. As $G$ is $\chi$-critical, $G^{\prime}=G$, and hence $T=T^{\prime}$. However, the complement of $G$ is connected and $C \backslash C^{\prime} \neq \emptyset$, thus $G_{0}$ must be an empty graph, i.e., $B=T=\emptyset$, and $a=|C|=1$. It follows that $G=G_{1}$ is a nontrivial basic graph.

As we have observed in the introduction, the degree sequence $D=(5 k-$ 
$3)^{5 k}$ has $\chi(D)=3 k$ (realized by the join of 5-cycles) and $\omega(D)=\left\lfloor\frac{5 k}{2}\right\rfloor$. Thus, $\chi(D)=\frac{6}{5} \omega(D)+\frac{3}{5}$ if $k$ is odd (and $\chi(D)=\frac{6}{5} \omega(D)$ if $k$ is even). Our next result shows that this example is the worst possible when comparing $\chi(D)$ and $\omega(D)$.

Theorem 3.2. For every graph $G, \chi(G) \leq \frac{6}{5} \omega(D(G))+\frac{3}{5}$.

Proof. Suppose for a contradiction that $G$ is a smallest counterexample, and let $n$ be the number of vertices of $G$. By Lemma 3.1, we may assume that $G$ is a join of basic graphs $G_{1}, G_{2}, \ldots, G_{k}$. Also,

$$
\chi(G)=\chi\left(G_{1}\right)+\chi\left(G_{2}+\cdots+G_{k}\right) \leq \chi\left(G_{1}\right)+\frac{6}{5} \omega\left(D\left(G_{2}+\cdots+G_{k}\right)\right)+\frac{3}{5}
$$

and

$$
\omega(D(G)) \geq \omega\left(D\left(G_{1}\right)\right)+\omega\left(D\left(G_{2}+\cdots+G_{k}\right)\right) .
$$

Thus $\chi\left(G_{1}\right)>\frac{6}{5} \omega\left(D\left(G_{1}\right)\right)$, and by symmetry, $\chi\left(G_{i}\right)>\frac{6}{5} \omega\left(D\left(G_{i}\right)\right)$ and hence $G_{i}$ is nontrivial for $1 \leq i \leq k$. Let $n_{i}=2 m_{i}+1$ be the number of vertices of $G_{i}$. As $G_{i}$ is basic, $\chi\left(G_{i}\right)=m_{i}+1$ and $\omega\left(D\left(G_{i}\right)\right)=m_{i}$. Note that the smallest nontrivial basic graph $C_{5}$ has $n_{i}=5$, thus $\chi(G)=\frac{n+k}{2} \leq \frac{3 n}{5}$. On the other hand, $\delta(G)=\min \left\{n-n_{i}+\delta\left(G_{i}\right): 1 \leq i \leq k\right\} \geq \min \left\{n-m_{i}-1\right.$ : $1 \leq i \leq k\} \geq \frac{n-1}{2}$, and hence by Theorem 2.7, $\omega(D(G)) \geq \frac{n-1}{2}$, and $\chi(G) \leq \frac{3 n}{5}=\frac{6(n-1) / 2+3}{5} \leq \frac{6}{5} \omega(D(G))+\frac{3}{5}$.

The chromatic number of any graph $G$ satisfies the following trivial bounds:

$$
\omega(G) \leq \chi(G) \leq \Delta(G)+1 .
$$

Reed investigated general bounds on the chromatic number that can be expressed as a convex combination of $\Delta(G)$ and $\omega(G)$. He proposed the following

Conjecture 3.3 (Reed [7]). $\chi(G) \leq\left\lceil\frac{1}{2}(\omega(G)+\Delta(G)+1)\right\rceil$ for every graph $G$.

For degree sequences, we prove the following stronger bound of the same form:

Theorem 3.4. For every graph $G, \chi(G) \leq \frac{4}{5} \omega(D(G))+\frac{1}{5} \Delta(G)+1$.

Proof. By considering a smallest counterexample, we see that $G$ is $\chi$-critical. By Lemma 3.1 we conclude that $G$ is a join of basic graphs $G_{1}, \ldots, G_{k}$. Suppose first that $k=1$, i.e., $G$ is basic. As $G$ is $\chi$-critical, $\Delta(G) \geq$ 
$\delta(G) \geq \chi(G)-1$. As $G$ is basic, $\omega(D(G)) \geq \chi(G)-1$. We conclude that $\frac{4}{5} \omega(D(G))+\frac{1}{5} \Delta(G)+1 \geq \chi(G)$.

Suppose now that $k>1$. Let us first consider the case when one of the graphs $G_{i}$ in the join, say $G_{1}$, is trivial, i.e., $\chi\left(G_{1}\right) \leq \omega\left(D\left(G_{1}\right)\right)$. Observe that $\Delta\left(G_{1}\right) \geq \omega\left(D\left(G_{1}\right)\right)-1$, thus $\frac{4}{5} \omega\left(D\left(G_{1}\right)\right)+\frac{1}{5} \Delta\left(G_{1}\right) \geq \chi\left(G_{1}\right)-\frac{1}{5}$. Note that $\Delta(G) \geq \Delta\left(G_{1}\right)+\left|V\left(G_{2}+\cdots+G_{k}\right)\right| \geq \Delta\left(G_{1}\right)+\Delta\left(G_{2}+\cdots+G_{k}\right)+1$. It follows that $\chi(G)=\chi\left(G_{1}\right)+\chi\left(G_{2}+\cdots+G_{k}\right) \leq\left(\frac{4}{5} \omega\left(D\left(G_{1}\right)\right)+\frac{1}{5} \Delta\left(G_{1}\right)+\frac{1}{5}\right)+$ $\left(\frac{4}{5} \omega\left(D\left(G-G_{1}\right)\right)+\frac{1}{5}\left(\Delta(G)-\Delta\left(G_{1}\right)-1\right)+1\right) \leq \frac{4}{5} \omega(D(G))+\frac{1}{5} \Delta(G)+1$. This is in a contradiction with $G$ being a counterexample. It follows that each $G_{i}$ is a nontrivial basic graph.

Let $\left|V\left(G_{i}\right)\right|=2 m_{i}+1$, let $m=\min \left\{m_{i} \mid 1 \leq i \leq k\right\}$, and observe that $m \geq 2$. We may assume that $m=m_{1}$. Clearly, $\Delta=\Delta(G) \geq \Delta\left(G_{1}\right)+n-$ $(2 m+1) \geq n-m-1$, where $n=|V(G)|$. As in the proof of Theorem 3.2 we conclude that $\omega=\omega(D(G)) \geq \frac{n-1}{2}$.

¿From the facts that $(m-2)(k-1) \geq 0$ and $n \geq(2 m+1) k$, it follows that $2 m+5 k-n+6 \leq 10$. Now,

$$
\begin{aligned}
\chi(G) & =\sum_{i=1}^{k} \chi\left(G_{i}\right)=\sum_{i=1}^{k}\left(m_{i}+1\right)=\frac{1}{2} n+\frac{1}{2} k \\
& =\frac{4}{5}\left(\frac{n-1}{2}\right)+\frac{1}{5}\left(\frac{n-1}{2}\right)+\frac{k+1}{2} \\
& \leq \frac{4}{5} \omega+\frac{1}{5}(n-m-1)+\frac{1}{10}(2 m+5 k-n+6) \\
& \leq \frac{4}{5} \omega+\frac{1}{5} \Delta+1 .
\end{aligned}
$$

This is in a contradiction with $G$ being a counterexample, an the proof is complete.

Let us observe that the bound of Theorem 3.4 is tight for the degree sequences $D=(5 k-3)^{5 k}(k \geq 1)$. The same examples also show that the bound is best possible in the very strong sense as stated in the introduction.

The proofs of Theorems 3.2 and 3.4 can be combined to obtain the following stronger result.

Theorem 3.5. Let $D$ be a graphic degree sequence. There exists a realization $G \in \mathcal{R}(D)$ with the following properties:

(a) $\omega(G) \geq \frac{5}{6} \chi(D)-\frac{1}{2}$, and

(b) $\omega(G) \geq \frac{5}{4} \chi(D)-\frac{1}{4} \Delta(D)-\frac{5}{4}$. 


\section{The Hajós Conjecture for degree sequences}

The Hajós variation, Conjecture 1.3, is true in a quite strong sense. Let $h_{1}(G)=r$ if $r$ is the largest integer such that $G$ contains a subgraph obtained from $K_{r}$ by first selecting vertex-disjoint subgraphs $S_{1}, \ldots, S_{a}$ of $K_{r}$, where each $S_{i}$ is isomorphic to a star $K_{1, n_{i}}(1 \leq i \leq a)$, and then subdividing each edge of these stars exactly once.

Theorem 4.1. For every graph $G, \chi(G) \leq h_{1}(D(G))$.

Proof. Since $h_{1}(D(G)) \geq \omega(D(G))$ and $h_{1}\left(G_{1}+G_{2}\right) \geq h_{1}\left(G_{1}\right)+h_{1}\left(G_{2}\right)$, Lemma 3.1 shows that we can assume that $G$ is a nontrivial basic graph. Let $n=2 m+1$ be the number of vertices of $G$, and let $d_{1} \geq d_{2} \geq \cdots \geq d_{2 m+1}$ be the degree sequence of $G$. Let

$$
\alpha=\sum_{i=1}^{m}\left(d_{i}-d_{m+1}\right) \quad \text { and } \quad \beta=\sum_{i=m+2}^{2 m+1}\left(d_{m+1}-d_{i}\right) .
$$

As $\omega(D(G))<m+1$ and $\delta(G) \geq \chi(G)-1=m$, Lemma 2.8 implies that $\alpha+\beta<2 m-d_{m+1}$.

For $1 \leq i \leq m$, let $a_{i}=d_{i}-(m-1)$. Let $R=\frac{1}{2}\left(2 m-d_{m+1}+\alpha+\beta\right)$. Note that $R$ is an integer and $0 \leq \beta<R \leq 2 m-d_{m+1}-1 \leq m-1$. Since $\beta<R$, it follows that $d_{2 m-R}=d_{m+1}$. Let $t_{i}=d_{m+1}-d_{2 m-R+i}+1$ for $1 \leq i \leq R$ and $t_{R+1}=d_{m+1}-d_{2 m+1}+R-\beta$. An easy calculation shows that $\sum_{i=1}^{R+1} t_{i}=2 R$. All the numbers $t_{i}$ are positive, thus by Lemma 2.1, there exists a tree $T_{1}$ with vertices $v_{2 m-R+1}, v_{2 m-R+2}, \ldots, v_{2 m+1}$ such that the degree of $v_{2 m-R+i}$ in $T_{1}$ is $t_{i}$ for $i=1, \ldots, R+1$.

Our goal is to form a graph $G^{\prime}$ with the degree sequence $D(G)$ on vertices $v_{1}, v_{2}, \ldots, v_{2 m+1}$ in the following way. We start with a graph $H_{1}$ consisting of a union of two cliques, one on the vertex set $A=\left\{v_{1}, v_{2}, \ldots, v_{m}\right\}$ and the other one on the vertices $B=\left\{v_{m+1}, v_{m+2}, \ldots, v_{2 m+1}\right\}$. Next, we shall delete the edges of $T_{1}$ or a slight modification of $T_{1}$. Finally, we shall add edges between $A$ and $B$ so that the degrees will be as requested. The modification of $T_{1}$ will be designed in such a way as to enable us to get the required subdivision of the $(m+1)$-clique, whose vertices of degree $m$ will be $v_{1}, \ldots, v_{m+1}$, and the only subdivided edges will be some of the edges incident with $v_{m+1}$.

Let us first consider the case when $d_{m+1} \geq m+\alpha$. Then $m+R-$ $\beta \leq 2 m-R$. Let $T_{2}$ be a forest obtained from $T_{1}$ by choosing $R-\beta-1$ neighbors of $v_{2 m+1}$, removing the edges joining them to $v_{2 m+1}$, and adding a matching between them and the vertices $v_{m+2}, v_{m+3}, \ldots, v_{m+R-\beta}$. Let 
$H_{2}=H_{1}-E\left(T_{2}\right)$. In order to get a graph $G^{\prime}$ whose degree sequence is $D(G)$, we have to add edges between $A$ and $B$. This has to be done in such a way that each vertex $v_{i} \in A$ is incident with $a_{i}$ added edges $(1 \leq i \leq m)$, and each vertex $v_{m+i} \in B(1 \leq i \leq m+1)$ is incident with $b_{i}$ edges, where $b_{i}$ is as follows. For $i=1$ and for $R-\beta+1 \leq i \leq m-R$, we have $b_{i}=d_{m+1}-m$, and for $2 \leq i \leq R-\beta$ and for $m-R+1 \leq i \leq m+1$, we have $b_{i}=d_{m+1}-m+1$. Note that

$$
\sum_{i=1}^{m} a_{i}=\sum_{i=1}^{m}\left(d_{i}-m+1\right)=\alpha+m\left(d_{m+1}-m+1\right)
$$

and

$$
\begin{aligned}
\sum_{i=1}^{m+1} b_{i} & =\left(d_{m+1}-m\right)(m+1)+R-\beta-1+R+1 \\
& =\alpha+m\left(d_{m+1}-m+1\right)=\sum_{i=1}^{m} a_{i} .
\end{aligned}
$$

Thus we can apply Lemma 2.3. (This is obvious if $d_{m+1}>m$; if $d_{m+1}=m$, then $\alpha=0$ by the assumption that $d_{m+1} \geq m+\alpha$; moreover, $\beta=0$ since $d_{i} \geq m$ for every $i$; thus $a_{i}=1$ for $i=1, \ldots, m$ and $b_{i}=1$ for $i=2, \ldots, m+1$, so Lemma 2.3 can be applied trivially for the sequence $b_{2}, b_{3}, \ldots, b_{m+1}$.) We conclude that there exists a bipartite graph $H_{3}$ with parts $A=\left\{v_{1}, v_{2}, \ldots, v_{m}\right\}$ and $B=\left\{v_{m+1}, v_{m+2}, \ldots, v_{2 m+1}\right\}$ such that the degree of $v_{i}$ is $a_{i}$ for $1 \leq i \leq m$ and the degree of $v_{m+i}$ is $b_{i}$ for $1 \leq i \leq m+1$, and $H_{3}$ has a matching $M$ covering $A$.

Let $G^{\prime}=H_{2} \cup H_{3}$. Observe that $D\left(G^{\prime}\right)=D(G)$ and $v_{m+1}$ is adjacent to all vertices in $B \backslash\left\{v_{m+1}\right\}$. Together with the matching $M$, this gives a possibility to join $v_{m+1}$ with all vertices $v_{1}, \ldots, v_{m}$ by using disjoint paths of length two. This shows that $G^{\prime}$ contains a subdivision of $K_{m+1}$, whose vertices of degree $m$ are $v_{1}, \ldots, v_{m+1}$. Each edge is subdivided at most once and all the subdivided edges are incident with $v_{m+1}$. Thus $h_{1}\left(G^{\prime}\right) \geq m+1$.

Suppose now that $d_{m+1} \leq m+\alpha-1$. Note that $\alpha>0$ in this case. Let $S$ be a set consisting of $v_{2 m+1}, R-\beta$ (arbitrarily chosen) neighbors of $v_{2 m+1}$ in $T_{1}$ and of vertices $v_{m+2}, v_{m+3}, \ldots, v_{2 m-R}$. Note that $|S|=m-\beta$, $R-\beta<|S|, 2(R-\beta)=2 m-d_{m+1}+\alpha-\beta \geq m-\beta+1>|S|$, and $|S| \geq m-\left(2 m-d_{m+1}-\alpha-1\right)=d_{m+1}-m+\alpha+1 \geq 2$. Thus by Lemma 2.4 there exists a graph $T_{3}$ with vertex set $S$ and $R-\beta$ edges such that $1 \leq \delta\left(T_{3}\right) \leq \Delta\left(T_{3}\right) \leq 2$. Let $S_{i} \subseteq S$ be the set of vertices of $T_{3}$ of degree $i$, for $i \in\{1,2\}$. Let $T_{4}$ be the graph obtained from $T_{1}$ by first removing the 
$R-\beta$ edges of $T_{1}$ induced by $S$ (i.e. those joining $v_{2 m+1}$ and the $R-\beta$ chosen neighbors), and then adding the $R-\beta$ edges of $T_{3}$. Let $H_{4}=H_{1}-E\left(T_{4}\right)$. As in the previous case, we let $b_{i}^{\prime}$ denote the number of edges that we need to add between $A$ and the vertex $v_{m+1+i}(i=1, \ldots, m)$ in order to form a graph whose degree sequences is $D(G)$. More precisely, let $b_{i}^{\prime}=d_{m+1}-m+1$ for all $i$ such that $1 \leq i \leq m$ and $v_{m+1+i} \notin S_{2}$, and $b_{i}^{\prime}=d_{m+1}-m+2$ for all $i$ such that $v_{m+1+i} \in S_{2}$. Let $a_{i}^{\prime}=a_{i}-1$ for $1 \leq i \leq d_{m+1}-m$ and $a_{i}^{\prime}=a_{i}$ for $d_{m+1}-m+1 \leq i \leq m$. If $a_{i}=1$ for some $i$, then we have $d_{m+1}=m$. This implies that $a_{i}^{\prime}>0$ for $1 \leq i \leq m$. By Lemma 2.3, there exists a bipartite graph $H_{5}$ with parts $A$ and $B \backslash\left\{v_{m+1}\right\}$ containing a perfect matching $M$, such that the degree of $v_{i}$ is $a_{i}^{\prime}$ for $1 \leq i \leq m$ and it is $b_{i-m-1}^{\prime}$ for $m+2 \leq i \leq 2 m+1$.

Let $G^{\prime}$ be the graph obtained from $H_{4} \cup H_{5}$ by adding edges between $v_{m+1}$ and $v_{1}, v_{2}, \ldots, v_{d_{m+1}-m}$. Again, we see that $D\left(G^{\prime}\right)=D(G)$ and $v_{m+1}$ is adjacent to all other vertices of $B$. Together with the matching $M$, this yields a subdivision of $K_{m+1}$, where each edge is subdivided at most once and all the subdivided edges are incident with $v_{m+1}$. We conclude that $h_{1}\left(G^{\prime}\right) \geq m+1$.

\section{References}

[1] C. Berge, Graphs and Hypergraphs, Second revised edition, NorthHolland and Elsevier, 1976.

[2] P. Catlin, Hajós' graph-coloring conjecture: variations and counterexamples, J. Combin. Theory Ser. B 26 (1979) 268-274.

[3] M. Chudnovsky, P. Seymour, The proof of Rao's conjecture on degree sequences, talk at the Banff workshop on Graph Minors, BIRS, Banff, Alberta, September 28 - October 3, 2008.

[4] P. Erdős, T. Gallai, Graphen mit Punkten vorgeschriebenen Grades, Mat. Lapok. 11 (1960) 264-274.

[5] A. R. Rao, The clique number of a graph with given degree sequence, in: A.R. Rao (Ed.), Proceedings of the Symposium on Graph Theory, MacMillan and Co. India Ltd., I.S.I. Lecture Notes Series, vol. 4, 1979, pp. 251-267.

[6] A. R. Rao, An Erdős-Gallai type result on the clique number of a realization of a degree sequence, unpublished. 
[7] B. Reed, $\omega, \Delta$, and $\chi$, J. Graph Theory 27 (1998) 177-212.

[8] N. Robertson, On Rao's Conjecture, a talk at the workshop Graph Theory, Oberwolfach, January 2005.

[9] N. Robertson and P. D. Seymour, Graph minors. XX. Wagner's Conjecture, J. Combin. Theory Ser. B 92 (2004) 325-357.

[10] N. Robertson, Z. Song, Hadwiger number and chromatic number for near regular degree sequences, J. Graph Theory, in press.

[11] C. Thomassen, Some remarks on Hajós' conjecture, J. Combin. Theory Ser. B 93 (2005) 95-105.

[12] C. Thomassen, Hajós' conjecture for line graphs, J. Combin. Theory Ser. B 97 (2007) 156-157.

[13] J.-H. Yin, J.-S. Li, Two sufficient conditions for a graphic sequence to have a realization with prescribed clique size, Discrete Math. 301 (2005) $218-227$. 